\title{
Final state correlations in open charm production at HERA
}

\author{
Gero Flucke* \\ (on behalf of the H1 and ZEUS collaborations) \\ DESY, D-22607 Hamburg, Germany \\ E-mail: flucke@mail.desy.de
}

\begin{abstract}
Open charm production in electron-proton $(e p)$ scattering at the high energies of HERA (centreof-mass energy $\sqrt{s} \sim 320 \mathrm{GeV}$ ) is an ideal tool to study perturbative Quantum Chromodynamics (pQCD), since the charm mass provides a hard scale.

The main charm production mechanism is photon-gluon fusion (PGF) where a photon emitted by the incoming electron interacts with a gluon in the proton forming a quark antiquark pair. The cross section is dominated by processes where the virtuality $Q^{2}$ of the exchanged photon is very small. The kinematic region of $Q^{2}<1 \mathrm{GeV}^{2}$ is generally referred to as photoproduction since the photon is almost real. In this regime resolved photon processes in which the photon auctuates into a hadronic state before the hard interaction, become important.

Investigating £nal state correlations increases the sensitivity to the details of the charm production mechanism. Here $\mathrm{H} 1$ and ZEUS measurements of $D^{*}+$ jet cross sections in photoproduction are presented. Comparing the pseudorapidity distributions of the $D^{*}$ and a jet not containing the $D^{*}$ reveals the presence of non-charm initiated jets. The difference in the azimuthal angle $\phi$ between the two highest $p_{t}$ jets shows the need for higher order contributions to describe the data.

Furthermore results from $\mathrm{H} 1$ investigations of the integrated jet shape in dijet events are shown, where charm is tagged by a muon. The observations contradict the Monte Carlo expectation of broad gluon initiated jets in a resolved enriched data sample.
\end{abstract}

International Europhysics Conference on High Energy Physics

July 21st - 27th 2005

Lisboa, Portugal

* Speaker. 
a)

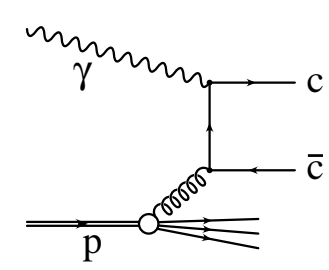

b)

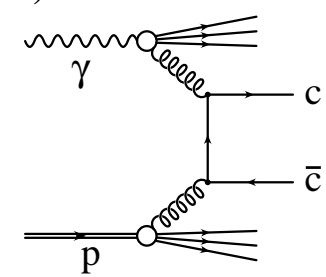

c)

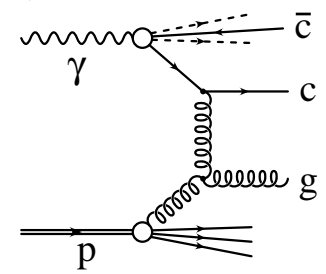

d)

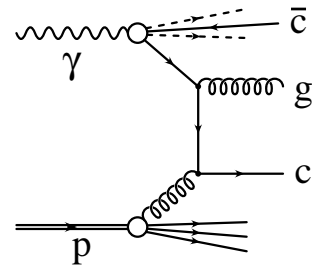

Figure 1: Leading order charm photoproduction: direct (a), resolved (b-d) and excitation processes (c-d).

\section{1. $D^{*}$ Jet Cross Sections}

Both $\mathrm{H} 1$ and ZEUS have measured jet cross sections in photoproduction where charm is tagged with $D^{*}$ mesons in the decay channel $D^{* \pm} \rightarrow D^{0} \pi_{s}^{ \pm} \rightarrow K^{\mp} \pi^{ \pm} \pi_{s}^{ \pm}$. The visible ranges of the H1 [1]

\begin{tabular}{l|c|c}
$D^{*}+\mathbf{j e t}(\mathbf{s})$ & H1 & ZEUS \\
\hline$Q^{2} /\left[\mathrm{GeV}^{2}\right]$ & $<0.01$ & $<1$ \\
$W_{\gamma p} /[\mathrm{GeV}]$ & & $130 . .280$ \\
$y$ & $0.29 . .0 .65$ & \\
\hline$p_{t}\left(D^{*}\right) /[\mathrm{GeV}]$ & $>2.0$ & $>3.0$ \\
$\left|\eta\left(D^{*}\right)\right|$ & $<1.5$ & $<1.5$ \\
\hline$p_{t}($ jet $) /[\mathrm{GeV}]$ & $>3.0$ & $>7.0(6.0)$ \\
$\eta($ jet $)$ & $-1.5 . .1 .5$ & $-1.5 . .2 .4$ \\
& $D^{*} \notin$ jet & $N($ jet $) \geq 2$
\end{tabular}
and ZEUS [2] measurements in $Q^{2}$, the inelasticity $y$ or the photon-proton centre-of-mass energy $W_{\gamma p}=\sqrt{y \cdot s}$, the transverse momentum $p_{t}$ and the pseudorapidity ${ }^{1} \eta$ of the $D^{*}$ and the jet(s) are summarised in the table on the left. H1 selects the highest transverse momentum jet not containing the $D^{*}$ meson, while ZEUS requires two jets where the $D^{*}$ is usually contained in one of the jets.

The cross sections as a function of the transverse momentum of the non- $D^{*}$-jet is shown in the Figs. $2 \mathrm{a}+\mathrm{d}$ (ZEUS) and $2 \mathrm{~g}+\mathrm{j}$ (H1) compared with next-to-leading (NLO) order QCD calculations in the massive (FMNR, [3]) or massless (ZMVFNS, [4]) scheme and with predictions from leading order calculations supplemented with parton showers and hadronisation, PYTHIA [5] and CASCADE [6]. The latter implements the $k_{t}$-factorising CCFM evolution equations whereas all other calculations follow the collinear DGLAP approach. All calculations reasonably describe the observed slope of the data.

For the $D^{*}+$ other jet measurement, cross sections as a function of the pseudorapidity of the $D^{*}$ and the jet are shown in the Figs. $2 \mathrm{~h}+\mathrm{k}$ and $2 \mathrm{i}+\mathrm{l}$. The $\eta\left(D^{*}\right)$ distribution falls steeply with increasing values of $\eta$ whereas $\eta$ (jet) is almost xat. For direct PGF processes (Fig. 1a) as simulated in PYTHiA (dir.), the $\eta$ spectra of the jet and the $D^{*}$ are found to be similar (cf. Fig. $2 \mathrm{k}+1$ ), showing that the difference is not caused by the cuts for the $D^{*}$ and the jet. Therefore the difference indicates the presence of non-charm initiated jets for $\eta>0$. All calculations include hard gluon emission diagrams (Fig. 1c) and hence can describe the observed shapes of $\eta\left(D^{*}\right)$ and $\eta$ (jet).

The difference in azimuthal angle $\Delta \phi^{j j}$ between the two highest $p_{t}$ jets in the event is sensitive to parton radiation in the plane transverse to the colliding photon and proton. The Figs. $2 \mathrm{~b}+\mathrm{e}(\mathrm{c}+\mathrm{f})$ show the distribution for a data sample enriched with events from direct (resolved) processes, i.e. with $x_{\gamma}^{o b s}>0.75\left(x_{\gamma}^{o b s}<0.75\right)$. Both distributions are rapidly falling from their maximum at $\Delta \phi^{j j}=\pi$. The NLO calculation underestimates these large tails, especially for the resolved enriched sample. In contrast, PYTHIA and especially HERWIG [7], both simulate higher order contributions with parton showers and describe the data fairly well.

\footnotetext{
${ }^{1} \eta=-\ln \tan (\theta / 2)$, where $\theta$ is the polar angle measured from the $z$-axis defned by the proton beam direction.
} 


\section{Jet Shapes}

The $D^{*}$ jet measurements have revealed the presence of non-charm initiated jets especially for $\eta>0$. H1 has investigated the nature of these jets by studying jet shapes [8]. A dijet sample is selected with the visible range listed in the table on the left where one of

H1 Jet Shape

\begin{tabular}{c}
\hline$Q^{2}<1 \mathrm{GeV}^{2}$ \\
$0.2<y<0.8$ \\
\hline $\mid \eta($ jet $) \mid<1.7$ \\
$p_{t}($ jet $)>7.0(6.0) \mathrm{GeV}$ \\
$N($ jet $) \geq 2$ \\
\hline$p_{t}(\mu)>2.5 \mathrm{GeV}$ \\
$35^{\circ}<\theta(\mu)<130^{\circ}$
\end{tabular}
the jets is tagged as charm jet using muons. The 'integrated jet shape' $\psi(r)=p_{t}^{j e t}(r) / p_{t}^{j e t}(R)$ is the fraction of the jet transverse momentum deposited within a cone of the radius $r=\sqrt{\Delta \eta^{2}+\Delta \phi^{2}}$ around the jet axis relative to the total transverse momentum of the jet within $R=1$, cf. Fig. $2 \mathrm{~m}$. Gluon initiated jets are expected to be broader compared to charm initiated jets and hence at a given radius the value of $\psi(r)$ is smaller.

Fig. $2 \mathrm{n}(\mathrm{o})$ shows the averaged integrated jet shape of the untagged jets for resolved (direct), $x_{\gamma}^{o b s}<0.75\left(x_{\gamma}^{o b s}>0.75\right)$, enriched events. The results are compared with a simulation of events generated by PYTHIA. The direct enriched sample is very well described by PYTHIA where the jets are mainly caused by the untagged (anti-)charm produced in PGF (Fig. 1a). The prediction for the resolved enriched sample shows broader jets than for the direct sample, in accordance with the expectation for a large contribution from gluon jets produced in charm excitation processes. But the data exhibit jets as narrow as for the direct sample and are not at all described by PYTHIA, contradicting the presence of a large fraction of gluon initiated jets.

\section{Summary}

Cross sections for photoproduction events with $D^{*}$ mesons and jets have been measured by $\mathrm{H} 1$ and ZEUS. Various pQCD approaches describe the $p_{t}$ and $\eta$ distributions where the latter reveal the presence of non-charm initiated jets for $\eta>0$. To describe the $\Delta \phi^{j j}$ distribution higher order contributions are needed, e.g. provided by parton showers. Investigating the integrated jet shape of non-charm tagged jets in charm dijet events shows discrepancies with the PYTHIA prediction. The large fraction of gluon jets predicted in a resolved enriched sample should lead to broader jets in contrast to the measurement. The details of charm photoproduction remain a challenge for theory.

\section{References}

[1] H1, Photoproduction of Events Containing a $D^{* \pm}$ Meson and a Jet at HERA, H1prelim-05-0732.

[2] S. Chekanov, et al. (ZEUS), Nucl. Phys., B729, (2005) 492 [hep-ex/ 0507089 ]

[3] S. Frixione, P. Nason, G. Ridolf, Nucl. Phys., B454, (1995) 3 [hep-ph/9506226].

[4] G. Heinrich, B. A. Kniehl, Phys. Rev., D70, (2004) 094035 [hep-ph/ 0409303 ].

[5] T. Sjöstrand, et al., Comput. Phys. Commun., 135, (2001) 238 [hep-ph/0010017] .

[6] H. Jung, Comput. Phys. Commun., 143, (2002) 100 [hep-ph/0109102] .

[7] G. Corcella, et al., HERWIG 6.3 Release Note (2001) [hep-ph/0107071] .

[8] H1, Study of Jet Shapes in Charm Photoproduction at HERA, H1prel im-05-077².

\footnotetext{
${ }^{2}$ available at https://www-h1.desy.de/publications/H1preliminary.short_list.html.
} 

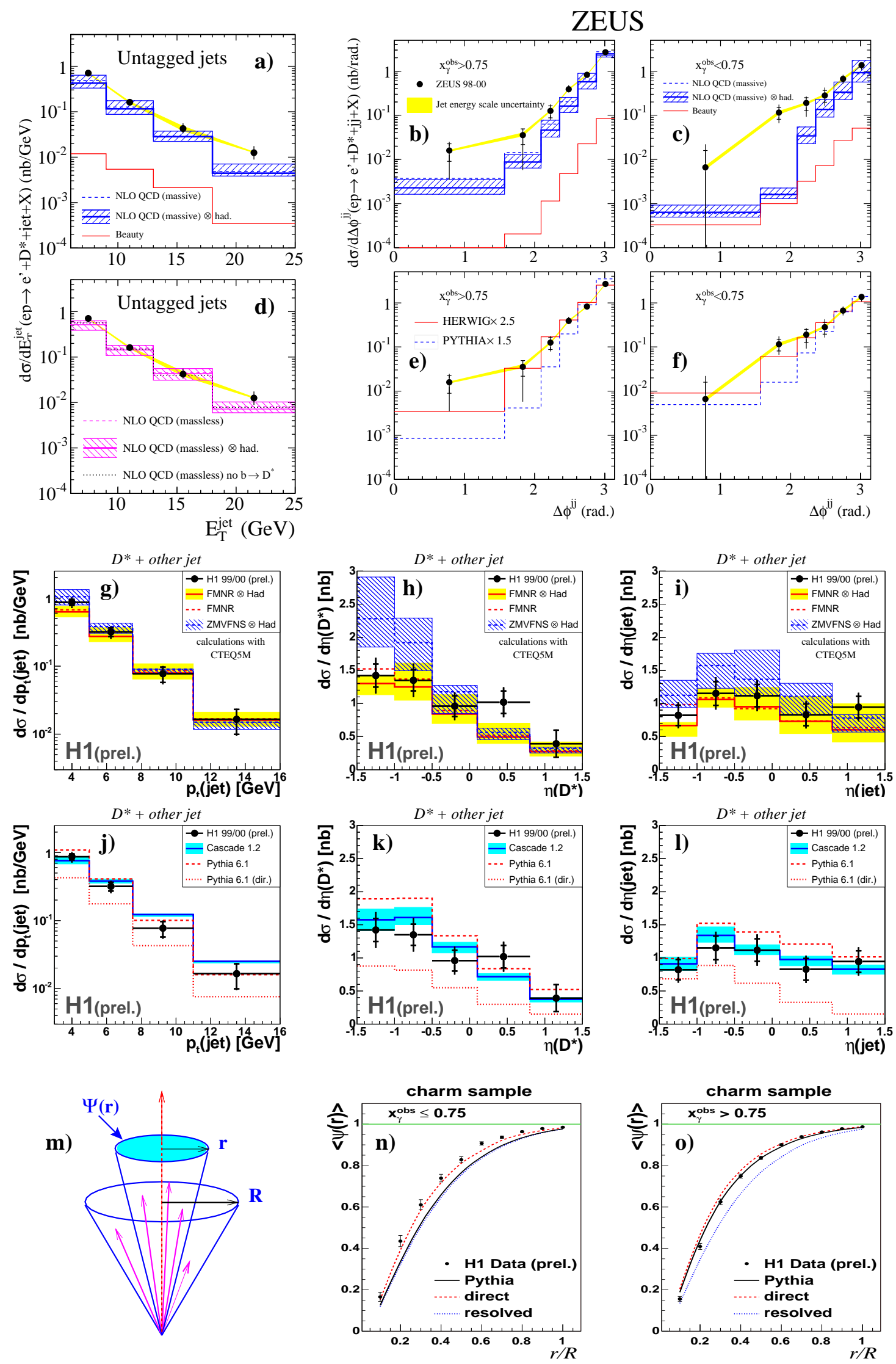

Figure 2: $D^{*}$ jet cross sections measured by ZEUS ( $D^{*}$ tagged dijets, a-f) and H1 $\left(D^{*}+\right.$ other jet, g-l) and integrated jet shapes for untagged jets in $\mu$-tagged charm dijet events (n-o). 